\title{
Charged Hadron Nuclear Modification Factors in the Beam Energy Scan from STAR
}

\author{
Stephen Horvat* (for the STAR collaboration) \\ Yale University \\ E-mail: stephen.horvateyale.edu
}

\begin{abstract}
Suppression of high transverse momentum $\left(p_{\mathrm{T}}\right)$ charged hadrons can be measured by the nuclear modification factor, which compares binary collision scaled $p_{\mathrm{T}}$ spectra from central heavy-ion collisions to a reference, either proton-proton $\left(R_{\mathrm{AA}}\right)$ or peripheral heavy-ion collisions $\left(R_{\mathrm{CP}}\right)$ by taking their ratio. At high $\sqrt{s_{\mathrm{NN}}}$ the nuclear modification factor at high $p_{\mathrm{T}}$ is observed to be suppressed, i.e. less than unity. The complex array of processes that can modify particle spectra in nuclear collisions span cold nuclear matter effects, a strongly interacting medium, and an extended phase of hadronic re-scatterings. RHIC's broad range of collision systems and energies combined with model comparisons provide the tools to investigate the beam energy dependence of nuclear modification factors and help to disentangle the spectral modifications that are due to each of these contributions. Measurements of charged hadron $R_{\mathrm{CP}}\left(\sqrt{s_{\mathrm{NN}}}, p_{\mathrm{T}}\right)$ for $\sqrt{s_{\mathrm{NN}}}=7.7$ $200 \mathrm{GeV}$ show a smooth transition from strong enhancement of high $p_{\mathrm{T}}$ charged hadrons at low energies to strong suppression at high energies. AMPT and HIJING failed to reproduce the results from data. Further tuning of the models may yet facilitate the investigation of the relative contributions from jet quenching and Cronin enhancement.
\end{abstract}

8th International Workshop on Critical Point and Onset of Deconfinement,

March 11 to 15, 2013

Napa, California, USA

\footnotetext{
* Speaker.
} 


\section{Introduction}

The energy loss of high momentum charged hadrons in central heavy-ion collisions at top energies at both the Relativistic Heavy Ion Collider (RHIC) and the Large Hadron Collider may be due to the formation of a Quark-Gluon Plasma (QGP), a strongly interacting medium with partonic degrees of freedom. The beam energy scan (BES) at RHIC is a program to collide gold ions over a broad range of energies to study the phase diagram for nuclear matter. The STAR experiment is using these data to try to determine at what beam energy key signatures for the formation of this strongly interacting medium of quarks and gluons disappear. One of these signatures is suppression in nuclear modification factors. Nuclear modification factors are used to compare central, small impact parameter, heavy-ion collisions with a reference. They reveal how the momentum distribution of particles produced in collisions differ between the two systems. In central heavy-ion collisions, where a QGP may be formed, high momentum particles produced early in the collision will subsequently traverse the medium and lose some of their momentum in the process [1]. In peripheral, large impact parameter, heavy-ion collisions and proton-proton $(p+p)$ collisions a QGP is unlikely to be produced making these collision systems good references. There is the nuclear modification factor where $p+p$ collisions are used as a reference,

$$
R_{\mathrm{AA}}=\frac{1}{\left(N_{\text {coll }}\right)_{\text {Central }}} \frac{\left(\frac{d^{2} N}{d p_{\mathrm{T}} d \eta}\right)_{\text {Central }}}{\left(\frac{d^{2} N}{d p_{\mathrm{T}} d \eta}\right)_{p+p}},
$$

and the nuclear modification factor where peripheral collisions are used as a reference,

$$
R_{\mathrm{CP}}=\frac{\left(N_{\text {coll }}\right)_{\text {Peripheral }}}{\left(N_{\text {coll }}\right)_{\text {Central }}} \frac{\left(\frac{d^{2} N}{d p_{\mathrm{T}} d \eta}\right)_{\text {Central }}}{\left(\frac{d^{2} N}{d p_{\mathrm{T}} d \eta}\right)_{\text {Peripheral }}} .
$$

Here $N_{\text {coll }}$ is the average number of binary collisions at a given impact parameter and can be estimated using a Glauber Monte Carlo [2]. If a heavy-ion collision were just a collection of $N_{\text {coll }}$ independent $p+p$-like collisions then both $R_{\mathrm{AA}}$ and $R_{\mathrm{CP}}$ would be unity for all kinetically accessible ranges of $p_{\mathrm{T}}$. Deviations from unity are due to nuclear modifications to the spectra. These modifications may result from cold nuclear matter (CNM) effects, from the formation of a QGP, and from final state interactions. CNM effects are modifications to the properties and interactions of nucleons when they are placed in nuclei [3]. They occur whether or not a medium is produced and can be measured and separated from QGP physics by studying collisions between light nuclei, like protons and deuterons, and heavy nuclei. This asymmetric collision system is unlikely to form a QGP, yet is sensitive to CNM effects. Among the CNM effects, the Cronin Effect is seen to be significant at high $p_{\mathrm{T}}$ in these asymmetric collisions [4]. The Cronin Effect is an experimentally observed enhancement of nuclear modification factors in asymmetric collisions. Some theorists have explained this enhancement as being due to multiple consecutive scatterings which can give a momentum boost to scattered partons, shifting their spectra toward higher $p_{\mathrm{T}}$ [5]. Whatever its source, this enhancement is a CNM effect which needs to be accounted for when looking for signatures for the formation of a QGP. High $p_{\mathrm{T}}$ partons (jets) generated in early hard scatterings and traversing a QGP would be expected to interact with the medium and lose energy. This medium induced jet quenching shifts the spectra toward lower $p_{\mathrm{T}}$, competing with the Cronin Effect. At 
and above $\sqrt{s_{\mathrm{NN}}}=62.4 \mathrm{GeV}$ nuclear modification factors have displayed suppression for high $p_{\mathrm{T}}$ charged hadrons [6], meaning that jet quenching is significantly stronger at these collision energies than the Cronin Effect.

A distinction is drawn between suppression and jet quenching where jet quenching refers to the physical process of jet energy loss in a medium while suppression refers to $R_{\mathrm{CP}}$ or $R_{\mathrm{AA}}$ being less than one. Measurements of $R_{\mathrm{CP}}\left(\sqrt{s_{\mathrm{NN}}}, p_{\mathrm{T}}\right)$ for $\sqrt{s_{\mathrm{NN}}}=7.7-200 \mathrm{GeV}$ are made in order to determine at which energy suppression turns off. Models are employed in order to perform the more difficult task of trying to determine the beam energy dependence of jet quenching.

\section{Method}

Data was gathered by the STAR detector in 2010-2011 from the RHIC BES (Table 1) [7]. Tracking is carried out using STAR's time projection chamber (TPC), a large acceptance detector with full azimuthal coverage and covering $|\eta|<1$. Single particle tracking efficiency is determined by the embedding method for $\pi^{ \pm}, K^{ \pm}, p$, and $\bar{p}$ for each dataset. Charged hadron efficiencies for each dataset are then constructed by combining the species-dependent efficiencies weighted by their spectra. Only those collisions whose vertices are reconstructed near the center of the detector are used. This ensures that the most efficient portion of the detector is utilized and reduces the possibility of collisions between ions and the beam pipe. Track quality cuts include requiring a minimum of 15 hits in the TPC and requiring that the reconstructed track pass within $1.5 \mathrm{~cm}$ of the reconstructed event vertex. Only tracks with $|\eta|<0.5$ are used in this analysis. The central events are the $5 \%$ of events with the highest multiplicity at mid-rapidity, while peripheral is defined here as 60-80\%. $N_{\text {coll }}$ is obtained from a Glauber Monte Carlo [2]. Efficiency corrected spectra histograms are filled in these centrality bins (Fig. 1) and their ratio, weighted by $N_{\text {coll }}$, gives $R_{\mathrm{CP}}$ (Fig. 2).

\begin{tabular}{cccl}
\hline \hline$\sqrt{s_{\mathrm{NN}}}(\mathrm{GeV})$ & $N_{\text {Events }}^{\mathrm{MB}}(\mathrm{M})$ & $N_{\text {Coll }}^{\text {Central }}$ & $N_{\text {Coll }}^{\text {Peripheral }}$ \\
\hline 7.7 & 4 & $773 \pm 28$ & $19.2 \pm 6.3$ \\
11.5 & 12 & $784 \pm 27$ & $19.1 \pm 7.8$ \\
19.6 & 36 & $800 \pm 27$ & $18.9 \pm 6.9$ \\
27 & 70 & $841 \pm 28$ & $20.0 \pm 8.6$ \\
39 & 130 & $853 \pm 27$ & $19.4 \pm 7.7$ \\
62.4 & 67 & $904 \pm 27$ & $19.3 \pm 7.7$
\end{tabular}

Table 1: STAR data from 2010-2011 for the BES. $N_{\mathrm{Events}}^{\mathrm{MB}}$ refers to the number of minimum bias events. The uncertainty on $N_{\text {Coll }}$ has contributions from the uncertainties in the $p+p$ scattering cross section, the Woods Saxon parameterization, and is dominated by the uncertainty in the total cross section.

\section{Results}

The tracking efficiency corrected spectra (Fig. 1) are smoothly falling with small statistical uncertainties. The slope of the peripheral spectra have a stronger $\sqrt{s_{\mathrm{NN}}}$ dependence than the central spectra do with the $7.7 \mathrm{GeV}$ data falling more steeply than the $62.4 \mathrm{GeV}$. The enhancement of the central spectra relative to the peripheral is revealed more clearly in the charged hadron 

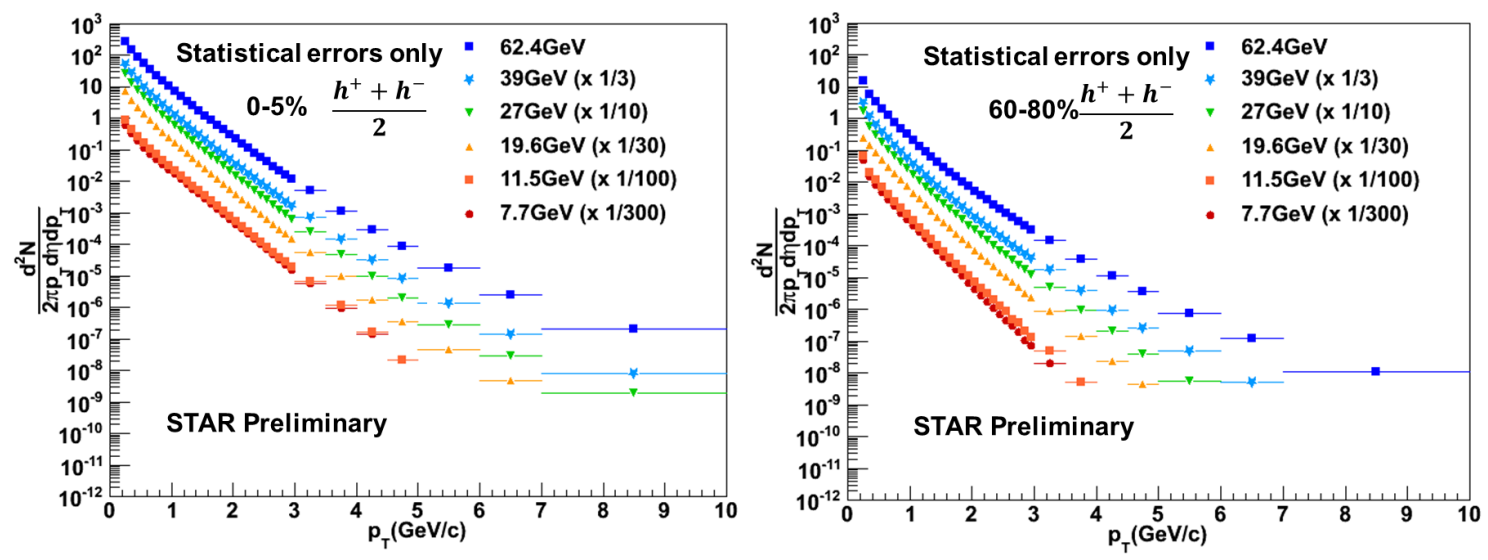

Figure 1: (Color online) Efficiency corrected charged hadron spectra for central (left) and peripheral (right) collisions for RHIC BES energies. $p_{\mathrm{T}}$ dependent errors are statistical only. The spectra are scaled for readability. The horizontal error bars indicate bin width.

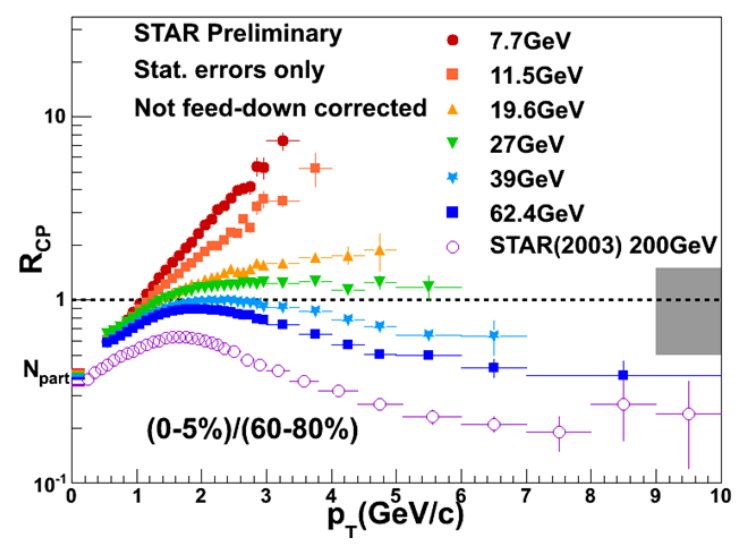

Figure 2: (Color online) Efficiency corrected charged hadron $R_{\mathrm{CP}}$ for RHIC BES energies. $p_{\mathrm{T}}$ dependent errors are statistical only. The gray error band comes from two sources added in quadrature. The first corresponds to the $p_{\mathrm{T}}$ independent uncertainty in $N_{\text {coll }}$ scaling. The second is a $20 \%$ deviation between 2003 STAR published, feeddown corrected, $R_{\mathrm{CP}}$ at $200 \mathrm{GeV}$ and the $R_{\mathrm{CP}}$ obtained in this analysis from the 2010 $200 \mathrm{GeV}$ data without feeddown corrections. The $N_{\text {part }}$ colored lines on the y-axis correspond to where unity would be for each energy if $N_{\text {part }}$ scaling were used rather than $N_{\text {bin }}$. The horizontal error bars indicate bin width.

$R_{\mathrm{CP}}\left(\sqrt{s_{\mathrm{NN}}}, p_{\mathrm{T}}\right)$ (Fig. 2) which reveals a smooth transition for high $p_{\mathrm{T}}$ hadrons from suppression at $\sqrt{s_{\mathrm{NN}}}=200 \mathrm{GeV}$, crossing unity near $27 \mathrm{GeV}$, up to a strong enhancement for $\sqrt{s_{\mathrm{NN}}}=7.7 \mathrm{GeV}$. These spectra are not feeddown corrected and the data points are shown with statistical uncertainties only. The $2010200 \mathrm{GeV}$ data were also studied in this analysis and the deviation between this new $R_{\mathrm{CP}}$ without feeddown corrections and the $R_{\mathrm{CP}}$ in STAR's 2003 paper [6] with feeddown corrections are quantified. The gray box corresponds to the systematic uncertainty on $N_{\text {coll }}$ added in quadrature with this $20 \%$ deviation. 

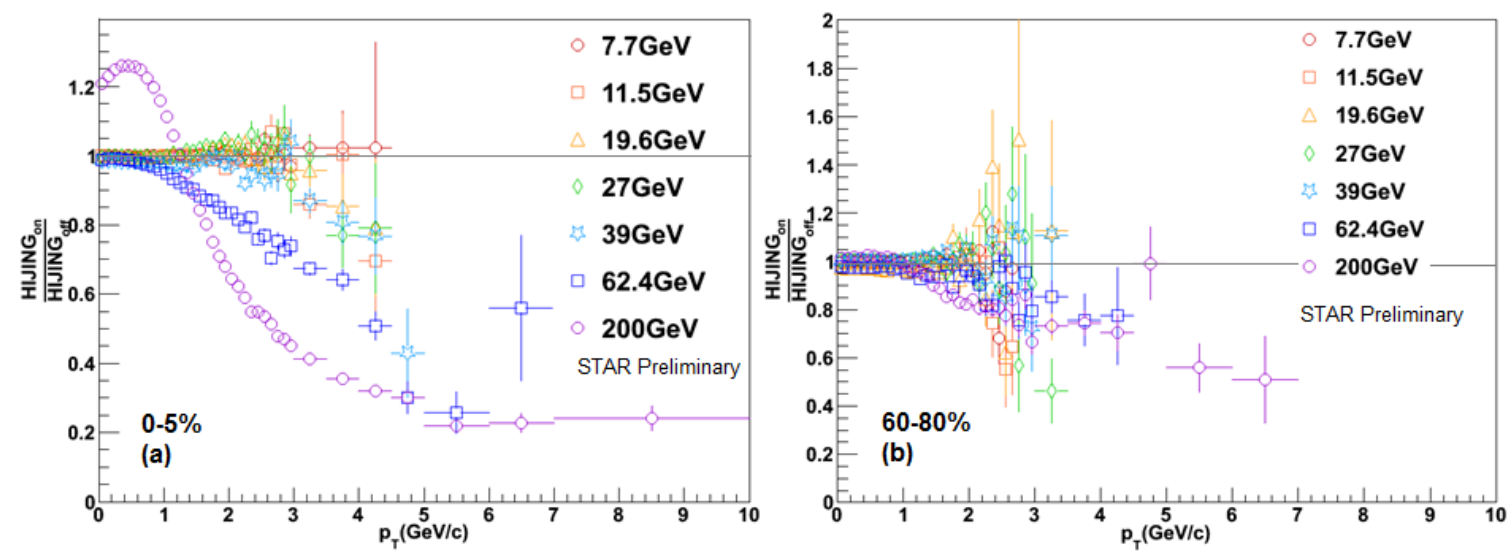

Figure 3: (Color online) The ratios of HIJING produced spectra with jet quenching on over HIJING produced spectra with jet quenching off are shown for central (a) and peripheral (b) collisions. $p_{\mathrm{T}}$ dependent errors are statistical only. The horizontal error bars indicate bin width.

\section{Models}

In order to understand the relative modification from the Cronin Effect and jet quenching we employ models where the different model elements can be turned on and off. This allows us to look at what the model would predict with or without jet quenching, for example. The two models employed here are the Heavy Ion Jet INteraction Generator (HIJING) [8] and A MultiPhase Transport model (AMPT) [9]. Only final state $\pi^{ \pm}, K^{ \pm}, p$, and $\bar{p}$ are studied. Centrality selection is carried out in the same way as for the data and the same values for $N_{\text {coll }}$ at each $\sqrt{s_{\mathrm{NN}}}$ are used. Again, we only take those tracks with $|\eta|<0.5$. Approximately one million events were produced for each model at each energy.

\subsection{HIJING}

HIJING 1.383 was used with default parameters and spectra for central and peripheral collisions were obtained. Ideally the ratio of these spectra to those from the data would be unity. Instead we find that the model fails to reproduce the data (Fig. 3). Model comparisons were also produced with jet quenching turned off in order to quantify the effects of this model component on the spectra. The ratio of the spectra with quenching on to that with quenching off is obtained for central and peripheral collisions (Fig. 4). There is little effect on peripheral collisions as would be expected. The strongest effects on central collisions are seen for the collision energies $62-200 \mathrm{GeV}$.

\subsection{AMPT}

Spectra for central and peripheral collisions were produced using AMPT v1.21/v2.21 set to its default parameters. AMPT with string melting off (v1.21), using the Lund String Fragmentation model for hadronization, did not capture the collision energy dependence of $R_{\mathrm{CP}}$. Neither string melting on (v2.21), which uses a coalescence model for hadronization, nor string melting off produced spectra that matched the data for either central or peripheral collisions (Fig. 5). 




Figure 4: (Color online) The ratios of HIJING produced spectra over experimentally collected charged hadron spectra for RHIC BES energies are shown with jet quenching turned on for central (a) and peripheral (b) centralities. These ratios are also shown for jet quenching turned off in central (c) and peripheral (d) collisions. $p_{\mathrm{T}}$ dependent errors are statistical only. The horizontal error bars indicate bin width.

\section{Summary}

The nuclear modification factor $R_{\mathrm{CP}}$ has been studied as a function of transverse momentum and collision energy. For high $p_{\mathrm{T}}$, a smooth transition from suppression to enhancement is observed with decreasing $\sqrt{s_{\mathrm{NN}}}$. The relative contributions from processes that lead to enhancement versus jet quenching are not determined by this analysis alone. The failure of either HIJING or AMPT to reproduce the data prevents conclusions from varying model elements for jet quenching, Cronin enhancement, and string melting being made. Analyses that would quantify the modification due to the Cronin Effect may be possible by examining the collision energy dependence of asymmetric collisions, collisions between heavy and light nuclei. These data could also provide additional constraints for the tuning of models. Additionally, the collision energy dependence of jet quenching may be measured using triggered dihadron correlations.

\section{References}

[1] Thoma M. 1991 Phys. Lett. B 273128 

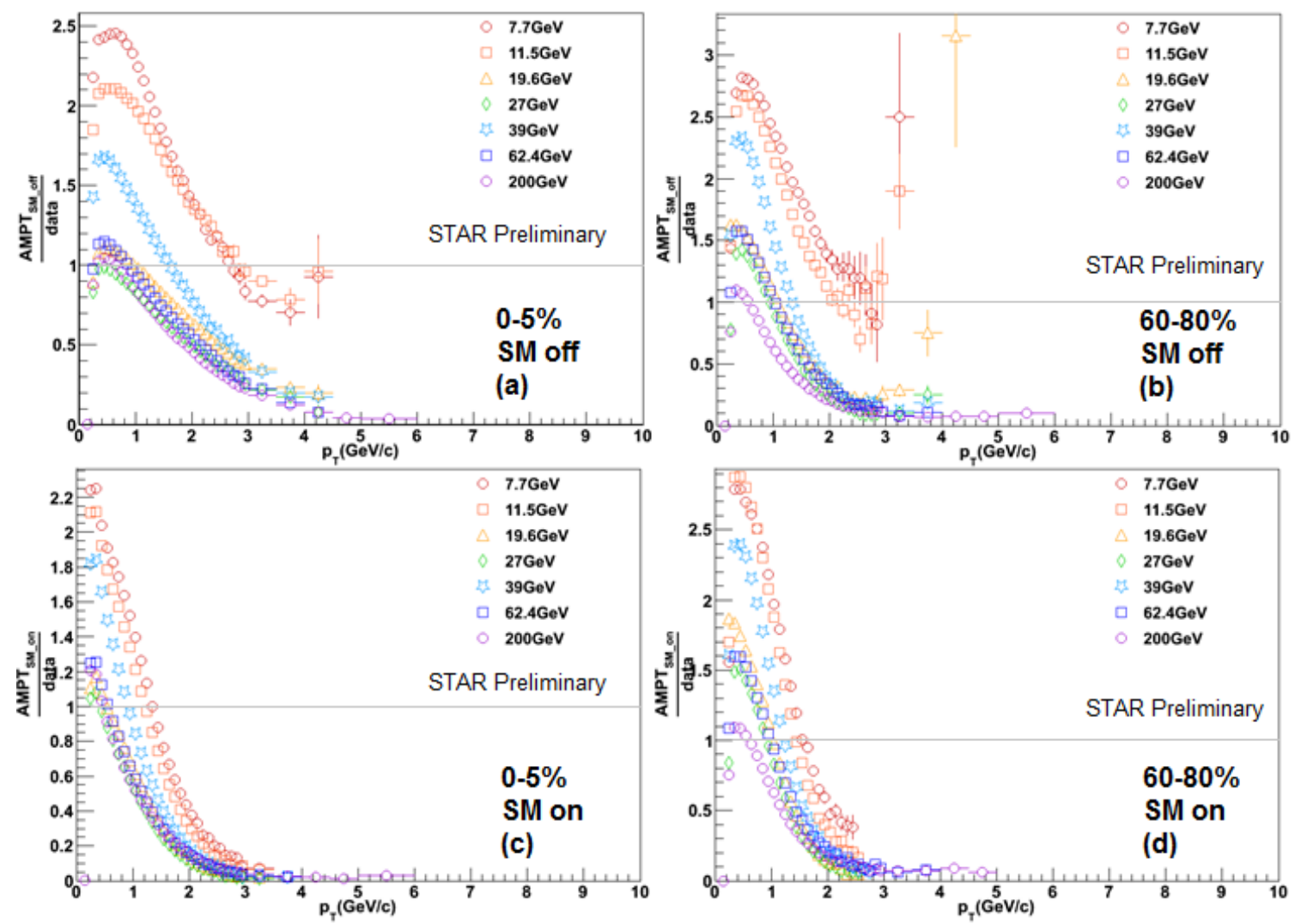

Figure 5: (Color online) The ratios of AMPT produced spectra over experimentally collected charged hadron spectra for RHIC BES energies are shown with string melting on for central (a) and peripheral (b) collisions. These ratios are also shown for string melting off in central (c) and peripheral (d) collisions. $p_{\mathrm{T}}$ dependent errors are statistical only. The horizontal error bars indicate bin width.

[2] Miller M. et al. 2007 Ann. Rev. of Nucl. and Part. Sci. 57205

[3] Kang Z., Vitev I., and Xing H. 2012 Phys. Lett. B 718482

[4] Cronin J.W. et al. 1975 Phys. Rev. D 113105

Adams J. et al. (STAR Collaboration) 2006 Phys. Lett. B 637161

[5] Kharzeev D., Kovchegov Y., and Tuchin K., 2003 Phys. Rev. D 68094013

[6] Adams J. et al. (STAR Collaboration) 2003 Phys. Rev. Lett. 91172302

Arsene I. et al. (BRAHMS Collaboration) 2005 Nucl. Phys. A 7571

Back B. B. et al. (PHOBOS Collaboration) 2005 Nucl. Phys. A 75728

Adcox K. et al., (PHENIX Collaboration) 2005 Nucl. Phys. A 757184

Abelev B. et al. (STAR Collaboration) 2007 Phys. Lett. B 655104

Aamodt K. et al. (ALICE Collaboration) 2011 Phys. Lett. B 69630

[7] Mohanty B. (for the STAR Collaboration ) 2011 J. Phys. G: Nucl. Part. Phys. 38124023

[8] Gyulassy M. and Wang X. 1994 Comput. Phys. Commun. 83307

[9] Zhang B. et al. 2000 Phys. Rev. C 61067901 